\title{
SOME OF THE CHALLENGES FACING SOCIETY AND SOCIAL PSYCHOLOGY
}

\author{
Yolanda Zografova \\ Institute for Population and Human Studies, Bulgarian Academy of Sciences, Bulgaria \\ E-mail: zografova@abv.bg
}

In the past year the international political and social situation changed significantly in Europe and its surrounding regions. The consequences are yet to unfold on a national and local level in terms of individual and societal notions and orientations within the complex events we are facing today. There is an increased number of displaced people due to dramatic events in their home countries, moving to Europe to seek refuge or better economic conditions. This has stimulated the emergence of attitudes, fears, representations within social groups and communities under various forms and often propagated and constructed by mass media, social networks and so on. Societies have gradually orientated towards accepting the diversity of ethnic groups, cultures and religions in the European countries. An increased number of citizens, however, including academics, cultural or other public figures oppose the coming of refugees and migrants and stand against politics of acceptance and recognition of incomers, both on a national and European level.

It is quite obvious, from a social-psychological perspective, that a social setting including intergroup contraposition on a macro-level, intergroup competition that is being often artificially maintained and that heightens the feelings of threat, may easily lead to rejection and hostility towards migrants and refugees. The step towards these attitudes results immediately in increased social distances.

In the social psychology knowledge, there are factors tested and proven by numerous studies and experiments that influence the development of inter-group tolerance and mutual acceptance. One of the most quoted and applied concepts is the Gordon Allport's Contact Theory. The researchers in this paradigm prove that the increase of the number and quality of contacts between different groups, including between representatives of the national majorities and those of the ethnic, migrant and other minorities, is a predictor of more positive perception and for reducing the prejudices. Of course, the relationship is neither as easy to apply, nor is possible without the presence of a number of factors, but it seems that the most important thing in the first place is the existence of real, positive social contacts.

For establishing conditions for positive contacts, however, the civil society should become active, opportunities should be provided by the responsible institutions, and European regulations should be certified on the acceptance of migrants and asylum seekers, etc. It is obvious that despite the huge possibilities of the web, social networks like Facebook, Twitter and others, despite the high-tech informative links, there is neither sufficient information nor clear concepts between the Europeans and for example people from the Middle East. In Europe, we do not know quite well the Tunisians, Lebanese, Syrians, their customs, traditions and religion. The opposite is also true - they hardly know well the European norms of behaviour and culture. Rather, this knowledge is gathered based on some media suggestions and on the social representations spread by way of interpersonal communications.

In the field of education many things could be realized in respect of the better study and understanding of other cultures, religions, lifestyles through the educational content. Textbooks should increasingly illustrate and talk about the similarities between peoples and the increased 
opportunities towards uniting the efforts of people of different cultures and skills. Encouraging the intercultural contacts from an early age, organizing various festivals, cultural exchanges between different communities within the country, for example between ethnic groups, and with representatives of other nations should be provoked.

Many other practices and knowledge could be distributed and exchanged among communities in Europe - both local and international. And there are no other good moves of the modern history unless people draw closer together, mutual knowledge and building a normative framework which sufficiently integrates the diversity of cultures, religions and customs.

Received: March 10, 2016

Accepted: May 15, 2016 